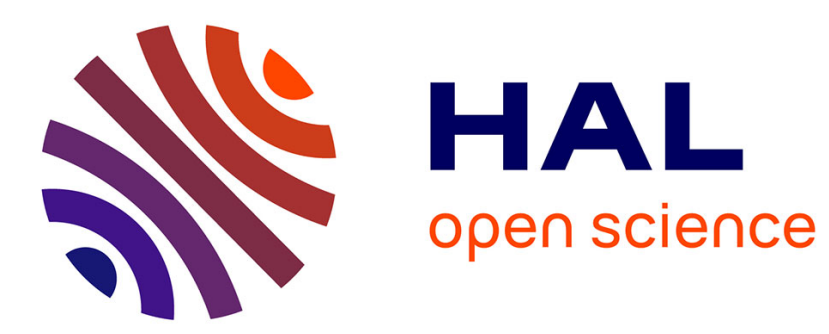

\title{
Acoustic inversions for measuring boundary layer suspended sediment processes
}

Peter Derreck Thorne, David Hurther, Ben Moate

\section{To cite this version:}

Peter Derreck Thorne, David Hurther, Ben Moate. Acoustic inversions for measuring boundary layer suspended sediment processes. Journal of the Acoustical Society of America, 2011, 130 (3), pp.11881200. 10.1121/1.3618728. hal-00619237

\section{HAL Id: hal-00619237 https://hal.science/hal-00619237}

Submitted on 29 Sep 2021

HAL is a multi-disciplinary open access archive for the deposit and dissemination of scientific research documents, whether they are published or not. The documents may come from teaching and research institutions in France or abroad, or from public or private research centers.
L'archive ouverte pluridisciplinaire HAL, est destinée au dépôt et à la diffusion de documents scientifiques de niveau recherche, publiés ou non, émanant des établissements d'enseignement et de recherche français ou étrangers, des laboratoires publics ou privés. 


\title{
Acoustic inversions for measuring boundary layer suspended sediment processes
}

\author{
Peter D. Thorne ${ }^{\text {a) }}$ \\ National Oceanography Centre, Joseph Proudman Building, 6 Brownlow Street, Liverpool, L3 5DA, \\ United Kingdom \\ David Hurther \\ Laboratory of Geophysical and Industrial Flows (LEGI), CNRS UMR 5519, Grenoble, France \\ Benjamin D. Moate \\ National Oceanography Centre, Joseph Proudman Building, 6 Brownlow Street, Liverpool, L3 5DA, \\ United Kingdom
}

(Received 25 August 2010; revised 25 May 2011; accepted 7 July 2011)

\begin{abstract}
Although sound has been applied to the study of sediment transport processes for a number of years, it is acknowledged that there are still problems in using the backscattered signal to measure suspended sediment parameters. In particular, when the attenuation due to the suspension becomes significant, the uncertainty associated with the variability in the scattering characteristics of the sediments in suspension can lead to inversion errors which accumulate as the sound propagates through the suspension. To study this attenuation propagation problem, numerical simulations and laboratory experiments have been used to assess the impact unpredictability in the scattering properties of the suspension has on the acoustically derived suspended sediments parameters. The results clearly show the commonly applied iterative implicit inversion can lead to calculated sediment parameters, which become increasingly erroneous with range, as the sound propagates through the suspension. To address this problem an alternative approach to the iterative implicit formulation is investigated using a recently described dual frequency inversion. This approach is not subject to the accumulation of errors and has an explicit solution. Here the dual frequency inversion is assessed and calculated suspended sediment parameters are compared with those obtained from the iterative implicit inversion. (C) 2011 Acoustical Society of America.
\end{abstract}

[DOI: $10.1121 / 1.3618728]$

PACS number(s): 43.30.Ft, 43.30.Gv, 43.30.Pc, 43.20.Fn [KGF] Pages: 1188-1200

\section{INTRODUCTION}

The use of megahertz sound to measure near bed boundary layer sediment transport processes has advanced significantly over the past two decades (Thorne and Hanes, 2002; Thorne and Bell, 2009). The boundary layer processes are due to dynamic interactions between the bedforms, the hydrodynamics and the mobile sediments, with each component having mutual feedback links. Acoustics has the capability to measure collocated and simultaneously the three components of this dynamic sediment triad. Multifrequency acoustic backscattering systems, ABS, are used to measure profiles of suspended particle size and concentration (Crawford and Hay, 1993; Thorne and Hardcastle, 1997), coherent acoustic Doppler velocity profilers, ADVP, are used to measure the three orthogonal components of flow (Zedel and Hay, 2002; Betteridge et al., 2006; Hurther and Lemmin 2008) and high resolution acoustic ripple profilers, ARP, are used to measure detailed changes in small scale bedforms (Williams et al., 2004; Traykovski, 2007; Cheel and Hay, 2008). The combined application of these acoustic technologies has made

\footnotetext{
a) Author to whom correspondence should be addressed. Electronic mail: pdt@pol. ac.uk
}

sound a valuable tool in the study of fundamental sediment transport processes (Hurther et al., 2011).

The application of sound to study sediment transport processes began with the measurement of suspended sediment concentration (Young et al., 1982; Hanes et al., 1988) and later suspended concentration and particle size (Hay and Sheng, 1992; Thorne and Hardcastle, 1997; Thorne et al., 2007). The technique uses a multifrequency transceiver system operating in the low megahertz region, $0.5-5.0 \mathrm{MHz}$, mounted about $1-2 \mathrm{~m}$ above the seabed and transmitting a short pulse of sound, $0.005-0.02 \mathrm{~m}$ in length. As the pulse propagates from the transceiver to the bed, the suspended sediments backscatter a portion of the sound and this is range gated to give a backscattered signal profile. The acoustic approach uniquely provides nonintrusively, high spatial-temporal resolution profiles of the suspended sediments referenced to the bed location (Vincent and Green, 1990; Lee et al., 2004; Dohmen-Janssen and Hanes, 2005). Such measurements are critical for the development and assessment of process based sediment transport models (Smyth et al., 2002; Davies and Thorne, 2005; van der Werf et al., 2008).

In this paper we revisit the use of acoustics for the measurement of suspended particle size and concentration and in particular assess the methodologies used to extract the suspended sediment parameters. To interpret the backscatter 
signal collected by ABS requires a description of the intrinsic scattering properties of the sediments in suspension, with the particle size probability distribution, to obtain the ensemble scattering characteristics. These scattering properties are used in the inversion to obtain the sediment parameters. The intrinsic scattering properties are dependent on the detailed shape, density and mineralogy of the sediments in suspension and the ensemble scattering characteristics vary with the form and width of the probability size distribution. Therefore to correctly interpret ABS data obtained at sea requires the intrinsic scattering properties of the sediments to be measured and to have a record of the time history of the form of the suspended sediment size distribution at each range bin over the deployment period. Generally this level of detail will not be available to most users of ABS systems and therefore users will necessarily employ generic sediment scattering expressions to analyze the acoustic data. The only sediment for which we have a generic scattering description at present is quartz sand. Therefore irrespective of whether the bed is quartz sand or other noncohesive sediments (e.g., carbonates, heavy minerals, lithic fragments) or a mixture, the inversion will use a generic model of the type proposed by Thorne and Meral 2008, based on a review of the scattering characteristics of quartz sands. Also unless there is knowledge to the contrary, the size distribution applied to the calculation of the ensemble scattering characteristics will be lognormal with an estimated distribution width, even though the size distribution may be normal, bimodal or some other function. Therefore normally in marine applications of $\mathrm{ABS}$, there are uncertainties associated with the scattering characteristics of the sediments in suspension

It is the profiling capability of acoustics which is one of its great advantages over more conventional instruments such as single height optical instruments (Hatcher et al., 2000); however, it is also the one that introduces the most difficulty in extracting the sediment parameters. The problem arises because at any particular range the backscattered signal is a function of the attenuation due to the suspension along the entire path through which the sound has propagated. This attenuation loss has to be accounted for in the calculation of the particle size and concentration profiles. Accounting for the loss generally leads to solutions based on inversion methodologies that use a successive iterative technique with an implicit equation along the path of propagation. In this way account is taken of the signal loss introduced by the suspended sediments themselves (Hanes et al., 1988; Thorne et al., 1991; Hay and Sheng, 1992; Thorne et al., 1993; Crawford and Hay, 1993). However, these successive iterations along the propagation path can be unstable, with small errors due to uncertainties in the acoustic suspension scattering characteristics, positively feeding back into the inversion and accumulating with range (Thorne et al., 1993; Thorne and Hardcastle, 1997; Vincent, 2007). Thus, at times of greatest interest, when there is substantial suspended concentration and transport, the attenuation by the suspended sediments is at its highest and under these conditions iterative implicit inversions can become less reliable due to imprecision in the representation of the sediment scattering characteristics. This leads to the sediment parame- ters derived from the acoustic data having greater uncertainties. (Hanes et al., 1988; Vincent et al., 1991; Hay and Sheng, 1992; Thorne et al., 1993; Thorne and Hardcastle, 1997; Vincent, 2007).

The problem of iterative implicit solutions, with associated attenuation positive feedback and instability, is common to a number of areas in physics where inverse techniques are used to quantify parameters associated with ensembles of scatterers. With regard to the use electromagnetic radiation for estimating precipitation rates of rain, snow and hail, numerous approaches have been adopted to account for attenuation due to the precipitation itself (Marzoug and Amayenc, 1994; Aydin et al., 1989; Bringi et al., 2001; Synder et al., 2010). The approaches are commonly based on empirical relations between the backscattered signal and total scattering cross section and often more recently the utilization of polarization effects to constrain the attenuation feedback. The approaches though of relevance to the present sediment problem do not readily translate to the acoustics field due to the specific empirical electromagnetic relationships employed and the lack of equivalence of polarization in acoustics. Closer to the field of the present study is the problem of measuring and accounting for the impact of the total scattering cross section (Foote, 1990; Foote, 1999; Zhao and Ona, 2003) on the acoustic estimation of fish stocks. In the fisheries studies (Toresen, 1991; Foote et al., 1992), as in some sediment studies (Thorne et al., 1995), the use of the bed echo has been utilized respectively to assess the impact of attenuation on the propagation of the sound through fish shoals and suspended sediments.

Focusing on suspended sediment studies and attempt to overcome the implicit, iterative and feedback problems, alternative inversions have been sought. Lee and Hanes (1995) developed a single frequency explicit technique without the need for iteration. The solution was a mathematical technique which was computationally more efficient, but had no greater insensitivity to inversion errors (Holdaway and Thorne, 1997). Thorne et al. (1995) suggested using the seabed echo to constrain an iterative implicit inversion for calculating concentration profiles when the particle size profile was invariant and known. Although the technique worked for the case studied, the general applicability of the approach remains uncertain. Shen and Lemmin $(1996,1998)$ developed an attenuation compensation approach based on having two separated transducers facing each other in an inline horizontal bistatic arrangement. The approach did improve the inversion stability; however, the arrangement is clearly not practical for sediment transport studies where vertical profiles are required, since one of the transducers would have to be located on the bed, thereby interfering with the sediment processes being measured. Thosteson and Hanes (1998) using a multifrequency approach, extended the explicit algorithm of Lee and Hanes (1995) and combined it with an implicit solution to yield profiles of particle size and concentration. However, as illustrated in the paper, the solution was not stable as attenuation increased.

Therefore at present, all the alternative inversion methodologies so far explored in the ABS literature are either no better, or indeed inferior, to the implicit iterative approach, or have a configuration which is inappropriate for vertically 
profiling suspended sediments. In the present study we evaluate the accuracy of the implicit iterative approach using numerical simulations and experimental measurements and present an alternative for the inversion of ABS data, based on a method developed by Hurther et al. (2011) referred to here as the dual frequency method. The inversion requires two frequencies and the algorithm is based on having a known invariant particle size distribution with range. Under this relatively restrictive particle size constraint an explicit equation with no requirement for iteration can be obtained and used to calculate suspended sediment concentration. In this formulation the concentration calculated at a particular range bin is independent of all other range bins. Such a solution becomes increasingly relevant as ABS utilize higher frequencies to increase spatial resolution, so they can probe high concentration seabed interface processes within the first few centimeters above the bed (Hurther and Thorne, 2011).

Here we explore and compare the impact that uncertainties in the scattering description of suspended sediments have on the implicit iterative and dual frequency inversion methods. Such uncertainties will almost invariably be present for field data due to variability in particle shape, particle mineralogy, and the suspended size distribution (Schaafsma and Hay, 1997; Thorne and Buckingham, 2004; Thorne and Meral, 2008). In this paper we also present an extension of the dual frequency method, which enables both particle size and concentration to be extracted, with improved accuracy compared to the traditional implicit iterative approach.

\section{BACKGROUND SCATTERING THEORY}

Under conditions of incoherent scattering the root-mean square backscattered voltage, $V$, from a suspension of particles with mass concentration, $C$, insonified with a piston transceiver, can be expressed as (Sheng and Hay, 1988; Thorne and Hanes, 2002)

$$
\begin{aligned}
& V=\left(\frac{K \mathfrak{R}}{r \Psi}\right) \sqrt{C} e^{-2\left(r \alpha_{w}+\alpha_{s}\right)}, \\
& K=\frac{f}{\left(\rho a_{c}\right)^{1 / 2}}, \quad \alpha_{s}=\int_{0}^{r} \xi C d r, \quad \xi=\frac{3 \chi}{4 \rho a_{c}}, \\
& f=\left[\frac{\int_{0}^{\infty} a n(a) d a \int_{0}^{\infty} a^{2} f_{i}(x)^{2} n(a) d a}{\int_{0}^{\infty} a^{3} n(a) d a}\right]^{1 / 2}, \\
& \chi=\frac{\int_{0}^{\infty} a n(a) d a \int_{0}^{\infty} a^{2} \chi_{i}(x) n(a) d a}{\int_{0}^{\infty} a^{3} n(a) d a}, \\
& a_{c}=\int_{0}^{\infty} a n(a) d a .
\end{aligned}
$$

The term $K$ represents the sediment backscattering properties, $\rho$ is the sediment grain density and $a_{c}$ is the mean particle radius. $r$ is the range from the transciever and $\psi$ accounts for the departure from spherical spreading within the transducer nearfield. $\mathfrak{R}$ is a system constant incorporating the transmit and receive sensitivity, the voltage transfer function for the system, the pulse length and the directivity function of the transceiver. $\alpha_{\omega}$ is the sound attenuation due to water absorption and $\alpha_{s}$ is the attenuation due to suspended sediment scattering. $f_{i}$ and $\chi_{i}$ are respectively the intrinsic form function and intrinsic normalized total scattering cross section for the particles in suspension and $x=k a$, where $k$ is the wavenumber and $a$ is the radius of the particles in suspension. Here intrinsic refers to the backscattering characteristics measured using suspensions sieved into narrow $1 / 4 \varphi$ size fractions which provide a nominally single particle size. $f$ and $\chi$ represent the ensemble mean scattering values obtained by integrating $f_{i}$ and $\chi_{i}$ over the probability size distribution of the particles in suspension, $n(a)$.

In the present study the recent formulations of Thorne and Meral (2008) were used to represent the intrinsic scattering properties of the suspended sediments, these are given in Eq. (2):

$$
\begin{aligned}
& f_{i o}=\frac{x^{2}\left(1-0.35 e^{-\left((x-1.5)^{2} / 0.7\right)}\right)\left(1+0.5 e^{-\left((x-1.8)^{2} / 2.2\right)}\right)}{1+0.9 x^{2}} \\
& \chi_{\text {io }}=\frac{0.29 x^{4}}{0.95+1.28 x^{2}+0.25 x^{4}} .
\end{aligned}
$$

To obtain $f$ and $\chi$ a size distribution for the suspension is required. Sediments are often categorized as having a mass size distribution, $m(a)$, which is lognormal (Soulsby, 1997), the form of which is given in Eq. (3):

$$
\begin{aligned}
& m(a)=\frac{1}{a \zeta \sqrt{2 \pi}} e^{-(\ln (a)-\kappa)^{2} / 2 \zeta^{2}} \\
& \zeta=\sqrt{\ln \left(\left(\sigma_{m} / a_{m}\right)^{2}+1\right)}, \quad \kappa=\ln \left(\frac{a_{m}^{2}}{\sqrt{a_{m}^{2}+\sigma_{m}^{2}}}\right) .
\end{aligned}
$$

In Eq. (3) $a_{m}$ and $\sigma_{m}$ are, respectively, the mean and standard deviation of the lognormal particle mass distribution of the sediment in suspension. For the acoustic analysis the distribution required is not $m(a)$, but $n(a)$, which is obtained from $3 m(a) / 4 \pi \rho a^{3}$ using an equivalent sphere. The form for $m(a)$, $n(a)$ and the impact the size distribution has on the intrinsic scattering characteristics is shown in Fig. 1(a) for a value of $\sigma_{m} / a_{m}=0.5$. Figure 1(a) shows $m(a)$ and $n(a)$ and as can be seen, the mean value of $n(a), a_{c}$, is approximately half the mean value of $m(a), a_{m}$. This has implication for sediment transport studies, because sedimentologists generally use the median grain diameter, $2 a_{50}$, based on $m(a)$, to designate the size of the particles in suspension. Therefore the radius relevant for the correct interpretation of acoustic backscatter data will have a value significantly different from $a_{50}$ for broad distributions of $m(a)$. Conversely, the value for the particle diameter resulting from acoustic inversions, $2 a_{c}$, will not be the $2 a_{50}$ generally used by sedimentologists. 

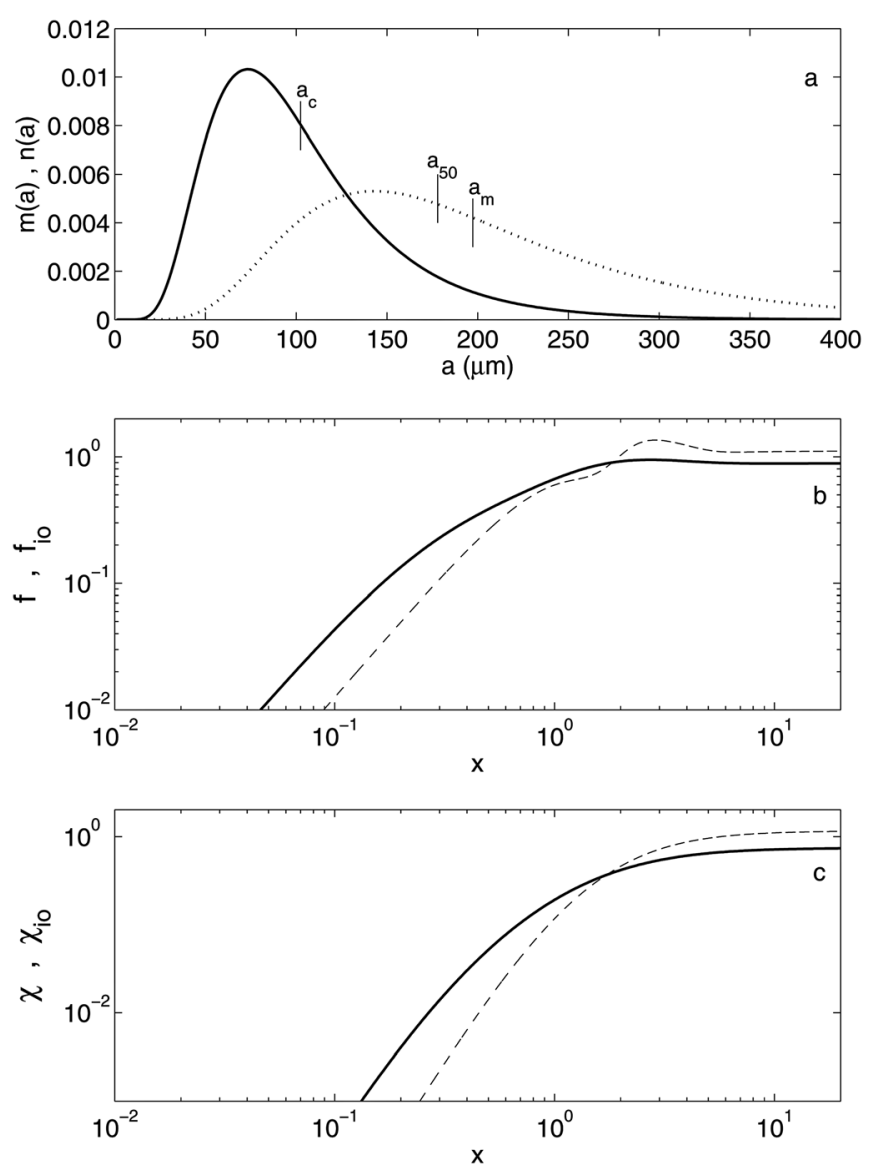

FIG. 1. (a) Lognormal mass distribution, $m(a)(\cdots)$, and particle size distribution, $n(a)(-)$, vs particle radius $a . a_{m}, a_{50}$, and $a_{c}$ are the radius respectively for the mean mass, median mass and mean particle size. (b) Form functions for the intrinsic scattering characteristics of the sediments $f_{i o}(--)$ and the ensemble $f(-)$ with $x$. (c) Normalized total scattering cross sections for the intrinsic scattering characteristics $\chi_{i o}(--)$ and the ensemble $\chi(-)$ with $x . x=k a$ for the intrinsic scattering and $x=k a_{c}$ for the ensemble scattering. $k$ is the wavenumber.

Figures 1(b) and 1(c) show that the introduction of a broad size distribution has a significant impact on the scattering characteristics of the suspended sediments. The ensemble values for $f$ and $\chi$ are plotted against $x=k a_{c}$ and relative to the intrinsic scattering characteristics of the particles, $f_{i o}$ and $\chi_{i o}$, they are increased significantly at low values of $x$, the Rayleigh regime, and reduced at high values of $x$, the geometric regime. Therefore since $n(a)$ [derived from $m(a)$ ] is an input parameter in the inversion and its representation impacts on the sediment scattering properties, the distribution used in the inversion can significantly alter the calculated profiles of particle size and concentration (Moate and Thorne, 2009).

For the simulations studied here it will be assumed that $m(a)$ is lognormal with $\sigma_{m} / a_{m}=0.5$ and Eqs. (2) forms the basis for the intrinsic scattering properties of the sediment in suspension. However, the expressions in Eq. (2) are generic for quartz sand, derived on the basis of fitting a single curve to a number of data sets. In practice the values of $f_{i}$ and $\chi_{i}$ for a particular ABS deployment are rarely known to a high degree of accuracy due to particle shape, mineralogy and density effects. Therefore to account for this variability in the suspended sediment intrinsic scattering characteristics, the calculations for the backscattered signal, $V$, in the scenar- ios addressed in the present study, used modified forms of $f_{i o}$ and $\chi_{i o}$, which were expressed as

$$
f_{i}=\frac{\beta\left(\beta x^{2}+1\right) f_{i o}}{\left(x^{2}+1\right)}, \quad \chi_{i}=\frac{\beta\left(\beta x^{2}+1\right) \chi_{i o}}{\left(x^{2}+1\right)} .
$$

The values used for $\beta$ were $0.8,1.0,1.2$ and represent the variability in the actual intrinsic scattering properties of suspensions expected to be encountered in different sandy environments. Equation (4) provided a differential scattering characteristic with $x$, which was greater in the geometric regime than in the Rayleigh regime. This is consistent with reported observations (Thorne and Buckingham, 2004). Figures 2(a) and 2(b) show the ensemble scattering values used in the calculations for $V$. The solid line was obtained using Eq. (4) with $\beta=1$ and the dotted and dashed lines were calculated using $\beta=1.2$ and $\beta=0.8$, respectively. The vertical lines in Fig. 2 indicate the location of the values for $x$ for the mean particle size and frequencies used in the simulated suspension inversions.

For all the simulated suspensions in the paper, the inversions were carried out with $\beta=1$, that is $f_{i}=f_{i o}$ and $\chi_{i}=\chi_{i o}$. For the calculation of $V$ three scenarios were adopted; (i) with $\beta=1$, this should yield the same inversion output concentration and particle size profiles as input, (ii) $\beta=0.8$, and (iii) $\beta=1.2$. For consistency with Fig. 2 all the inversion plots use a solid line for $\beta=1$, a dashed line for $\beta=0.8$, and a dotted line for $\beta=1.2$. It is the output and stability of the
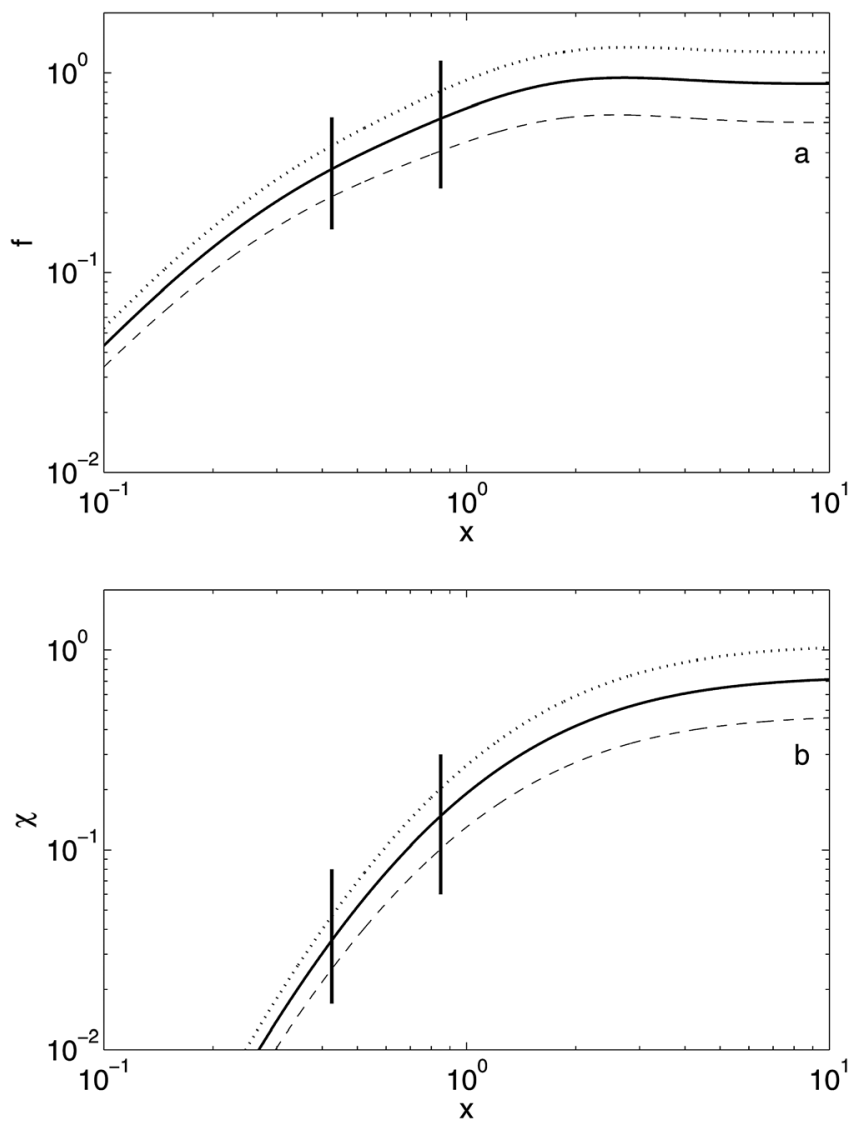

FIG. 2. Variation with $x$ of (a) the ensemble form function $f$ and (b) the ensemble normalized total scattering cross section $\chi$, for $\beta=0.8(--), 1.0(-)$, and $1.2(\cdots)$. The two vertical lines indicate the values of $x=k a_{c}$ used in the simulated suspension inversions. 
inversions, when sediment attenuation becomes significant, which is the focus of the present paper. This has been examined using scenarios where $V$ is enhanced or reduced, due to the variability in the suspension scattering characteristics which are represented here by the different values of $\beta$.

\section{PARTICLE SIZE AND CONCENTRATION PROFILES}

For the evaluation of Eq. (1) suspension parameters representative of the coastal marine environment needed to be defined. There is no general consensus on the form of the suspended sediment concentration profile with height above the bed, due to the various boundary layer mixing regimes. However, a common profile employed in sediment studies in coastal waters is an exponential (Nielsen, 1986; van der Werf et al., 2006) and the form used is given below

$$
C=C_{r} e^{-\left(z-z_{r}\right) / L} \text {. }
$$

For the mean particle size profile with height above the bed the expression used was (Thorne et al., 2002)

$$
a_{c}=a_{r}\left(\frac{z}{z_{r}}\right)^{-l} \text {. }
$$

Here $z$ is the height above the bed, $a_{r}$ and $C_{r}$ are the reference mean radius and concentration at height $z_{r}$ above the bed and $L$ and $l$ are lengths associated with boundary layer vertical mixing processes. The reduction in particle radius, $a_{c}$, with height above the bed is known to be much smaller than the reduction in $C$ (Crawford and Hay, 1993; Thorne et al., 2002). The concentration generally reduces by typically two orders of magnitude above a sandy bed, in the bottom 1-2 m, while the mean size reduction is more like $30 \%-50 \%$. Therefore in the first instance $a_{c}$ is assumed to be constant with $z$, $l=0$; this limitation will be examined later. The profiles for $a_{c}$ and $C$ used for the inversion simulations are respectively shown in Figs. 3(a) and 3(b). The reference was defined to be at $z_{r}=0.005 \mathrm{~m}$ and the mixing length for the concentration was chosen to be $L=0.2 \mathrm{~m}$ (Van Rijn, 1993). Equation (5) combined with the lognormal mass distribution in Eq. (3) and the scattering expressions in Eqs. (2) and (4) are considered to be reasonable representations of the suspended sediment conditions encountered in the nearshore coastal environment.

\section{INVERSION METHODOLOGIES AND RESULTS}

In practice $\mathrm{ABS}$ are deployed in coastal waters to measure suspended sediment profiles of particle size and concentration, therefore Eq. (1) is rearranged so that the acoustically derived suspended concentration, $M_{s}$, is the dependent variable

$$
M_{s}=\left(\frac{V r \Psi}{K \Re}\right)^{2} e^{4\left(r \alpha_{w}+\alpha_{s}\right)}
$$

This is referred to here as the inverse problem; that is the extraction of suspended sediment parameters from the backscattered acoustic signal, $V$. It is the impact the variability of
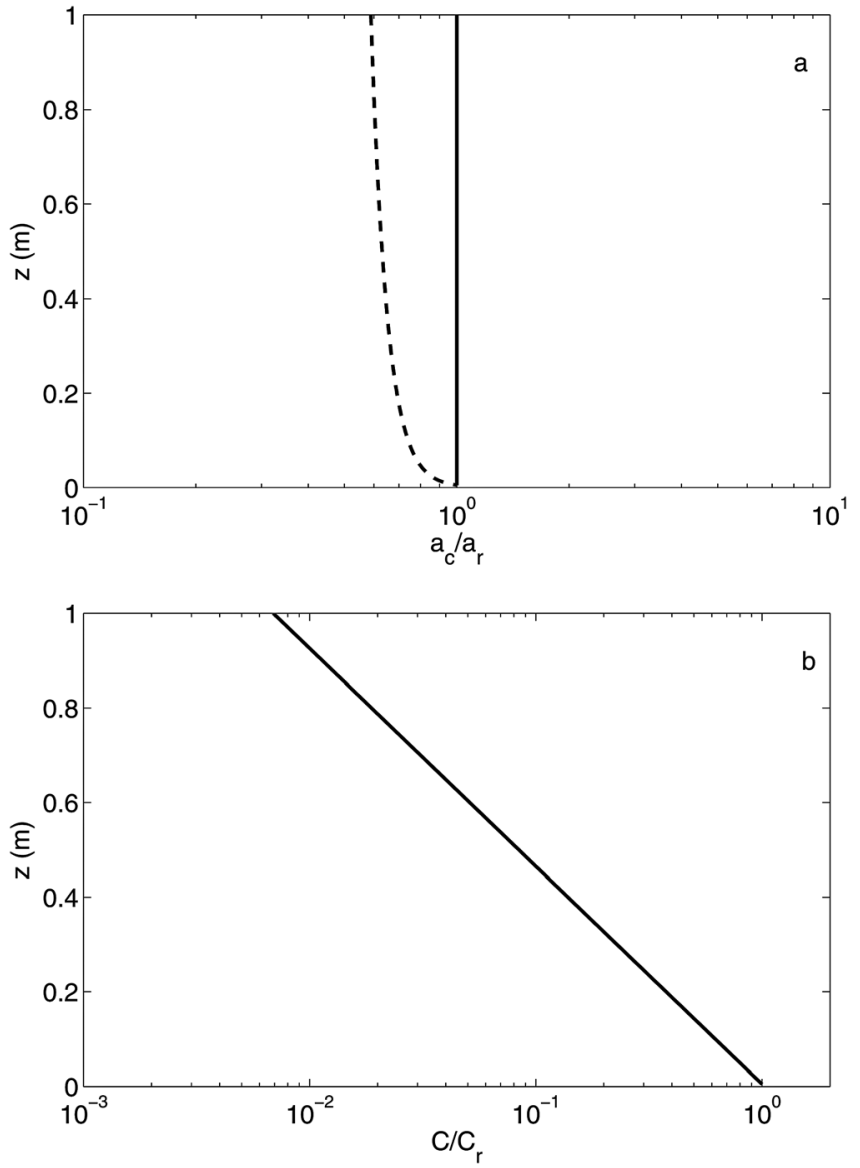

FIG. 3. Profiles of the variation with height above the bed, $z$, of (a) the normalized mean particle radius, $a_{c} / a_{r}$, for $l=0(-)$ and $l=0.1 \mathrm{~m} \mathrm{(--)}$ and (b) the normalized mass concentration, $C / C_{r}$.

the suspended sediment scattering characteristics have on the evaluation of Eq. (6), and the resulting profiles of $M_{s}$ or $M_{s}$ and $a_{s}$, the acoustically derived particle size, which is of interest in the present work. In the following simulations it is assumed that $\mathfrak{R}, \psi, \rho$, and $\alpha_{w}$ were known, the sediment scattering properties for the inversion were given by $f_{i o}, \chi_{i o}$ and $n(a)$ was derived from $m(a)$ which had a lognormal distribution with $\sigma / a_{m}=0.5$. The uncertainty in the inversion is due to the natural variability in $f_{i}$ and $\chi_{i}$ which is introduced into $V$ through changes in $\beta$. In the present study the $V$ profiles were calculated using 1.0 and $2.0 \mathrm{MHz}$, with $\beta=0.8,1.0,1.2$, $C_{r}=1.0,5.0,20 \mathrm{kgm}^{-3}$, and $a_{r}=100 \mu \mathrm{m}$. These were computed at $0.005 \mathrm{~m}$ range bin intervals and the range of operation was $1.0 \mathrm{~m}$; this range and resolution is typical of ABS systems. The results presented here are a subset of a much larger set of inversions and represent the generic salient features.

\section{A. Iterative implicit inversion}

The simplest and most straightforward inversion is under the special conditions that $a_{c}$ is known and $\alpha_{s}$ is negligible; the variation of $M_{s}$ with $r$ can then be readily evaluated using Eq. (6) and the suspended sediment concentration profile obtained. However, these criteria are not usually satisfied in dynamic coastal waters, instead the case where $\alpha_{s}$ cannot be ignored is of more general application. The second simplest case is if $a_{c}$ is constant with range 
and known and $\alpha_{s}$ is not negligible. Equation (6) now becomes implicit with $M_{s}$ being on either side of the equation. The equation is usually solved using an iterative methodology in which $\alpha_{s}$ is assumed initially to be zero and $M_{s o}$ is given by

$$
M_{s o}=\left(\frac{V r \Psi}{K \Re}\right)^{2} e^{4 r \alpha_{w}} .
$$

This provides an initial estimate for the concentration at the first range bin. An improved estimate can be obtained using

$$
M_{s 1}=M_{s 0} e^{4 \alpha_{s o}} \text {. }
$$

The value for $\alpha_{s o}$ was calculated using $M_{s o}$. In general Eq. (8) can be written as

$$
M_{s(j+1)}=M_{s 0} e^{4 \alpha_{s j}} .
$$

Equation (9) is iterated until a convergence criterion has been satisfied and the value for $M_{s}$ at the first range bin evaluated. The process is repeated sequentially stepwise at each range bin through the $V$ profile, with the accumulating sediment attenuation accounted for and the profile of $M_{s}$ with $r$ from the transducer progressively calculated. The difficulty with this methodology is that errors in the evaluation of $M_{s}$ feedback positively through $\alpha_{s}$ and accumulate with propagation range from the transducer.

This is illustrated in Fig. 4(a) using the $2.0 \mathrm{MHz} V$ profiles. For this inversion it is assumed $a_{c}$ is known and only $M_{s}$ is being estimated in the inversion. The different line types shown in Fig. 4(a) correspond with those in Fig. 2, with the dashed, solid and dotted lines respectively representing the calculation of $V$ using $\beta=0.8, \beta=1$, and $\beta=1.2$. As mentioned earlier all inversions were carried out using $\beta=1$, i.e., $f_{i}=f_{i o}$ and $\chi_{i}=\chi_{i o}$. Three reference concentrations were used, $C_{r}=1.0,5.0$, and $20.0 \mathrm{kgm}^{-3}$ in the calculations. As can be seen using the implicit iterative inversion when $\beta=1$, the original $C$ profile shown in Fig. 3(a) is recovered with $M_{s}=C$ for the three reference concentrations. For the case of $\beta=0.8$, the values for $f_{i}$ and $\chi_{i}$ are reduced relative to $f_{i o}$ and $\chi_{i o}$ and this generally leads to a reduction in the values of $V$, which in the iterative implicit inversion for $M_{s}$ results in an underestimate for $C$ that increases with range and reference concentration. For the case of $\beta=1.2$, the values for $f_{i}$ and $\chi_{i}$ are increased relative to $f_{i o}$ and $\chi_{i o}$ and this generally leads to an increase in the values of $V$, which in the iterative implicit inversion for $M_{s}$ results in an overestimate of $C$ that increases with range and reference concentration. Importantly, it can clearly be seen, that when $\beta \neq 1, M_{s}$ progressively diverges from $C$ with increasing range and reference concentration, due to the errors increasing as the inversion propagates along the profile of $V$ from the transducer to the bed. This is typical of the inherent positive feedback in the iterative implicit inversion as attenuation becomes a significant component of the backscattered signal.

To calculate suspended concentration, $M_{s}$ and particle size, $a_{s}$, a second acoustic frequency is needed. In the present
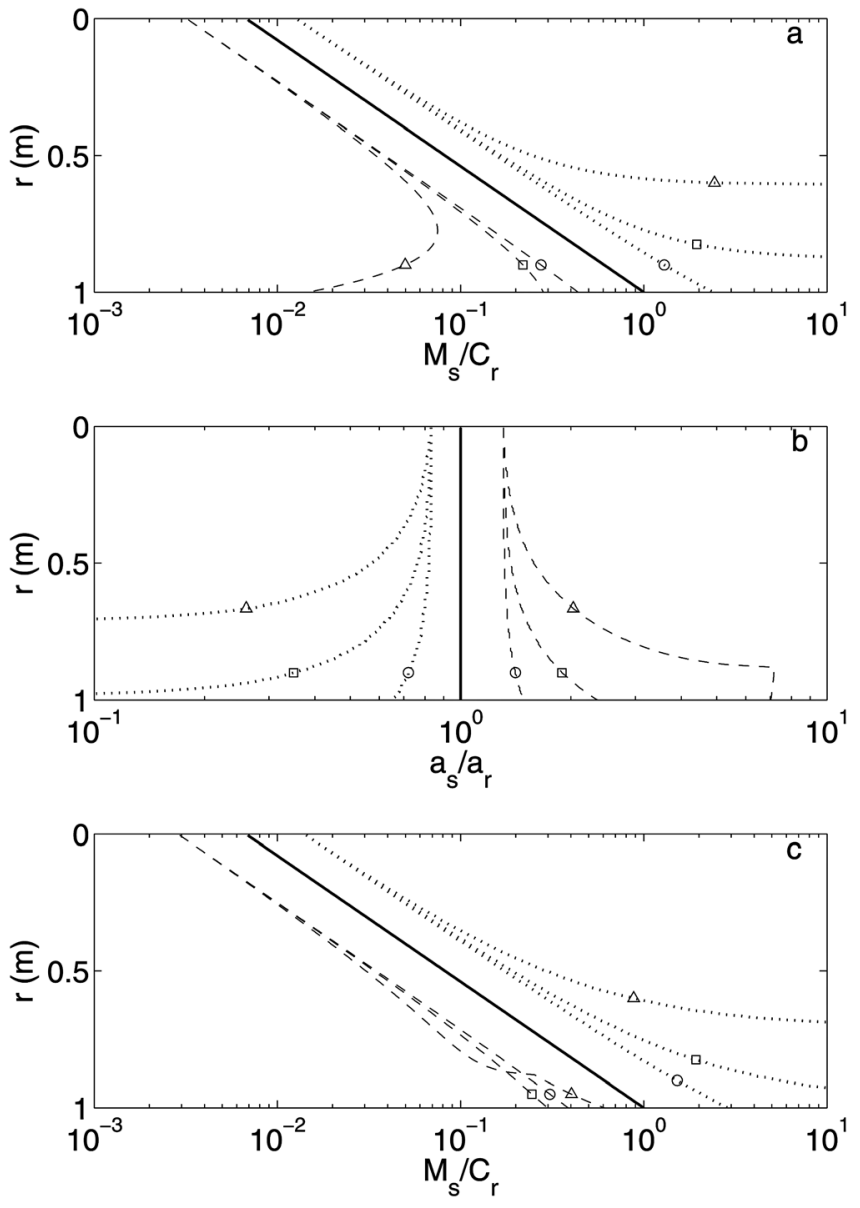

FIG. 4. Profiles from the iterative implicit inversion of the variation with range, $r$, of (a) normalized concentrations, $M_{s} / C_{r}$, when the particle size was range independent and known, (b) mean normalized particle size, $a_{s} / a_{r}$, and (c) normalized concentration, $M_{s} / C_{r}$, when both mean particle size and concentration were unknown. The three lines are for $\beta=0.8(--), 1.0(-)$, and $1.2(\cdots)$, the symbols represent $C_{r}=1.0(\bigcirc), 5.0(\square)$, and $20(\triangle) \mathrm{kgm}^{-3}$.

case 1.0 and $2.0 \mathrm{MHz}$ are used for illustration. These frequencies are typically used in $\mathrm{ABS}$. In the present inversion methodology $M_{s}$ was calculated for the first range bin at each frequency for a range of particle radii between 5-1000 $\mu \mathrm{m}$, using the same approach described in Eq. (9). A number of approaches have been used to obtain $a_{s}$ (Hay and Sheng, 1992; Crawford and Hay, 1993; Thostesen and Hanes, 1998) and the approach used here which was found to be robust (Thorne et al., 2007) was to calculate

$$
\begin{aligned}
& \Delta=\frac{\sigma_{c}}{M_{c}} \\
& M_{c}=\frac{1}{n} \sum_{i=1}^{n} M_{s i} \quad \sigma_{c}^{2}=\frac{1}{n} \sum_{i=1}^{n} M_{s i}^{2}-\left(\frac{1}{n} \sum_{i=1}^{n} M_{s i}\right)^{2}
\end{aligned}
$$

$M_{s i}$ is the concentration at each of the $n$ frequencies, two frequencies in the present study, and the minimum value in $\Delta$ over the size range 5-1000 $\mu \mathrm{m}$ was selected to obtain $a_{s}$ and $M_{s}$. The process was repeated sequentially stepwise at each range bin through the backscatter profile, with the accumulating sediment attenuation accounted for and the profile of $a_{s}$ and $M_{s}$ with $r$ from the transducer progressively 
calculated. The results are shown in Figs. 4(b) and 4(c). For $\beta=1$, the implicit iterative inversion yield $a_{s}=a_{c}$ and $M_{s}=C$. For $\beta=0.8, a_{s}$ overestimates $a_{c}$ and this consistently increases with range and concentration, although there is a slight modification to this trend for $r>0.9 \mathrm{~m}$ for $C_{r}=20$ $\mathrm{kgm}^{-3}$. For $M_{s}$ the values do not substantially increasingly diverge from $C$ with range, this is different to the case for $\beta=0.8$ in Fig. 4(a) and is due to the increased values for $a_{s}$ selected in the inversion procedure. For $\beta=1.2, a_{s}$ underestimate $a_{c}, M_{s}$ overestimate $C$ and both $\mathrm{a}_{\mathrm{s}}$ and $M_{s}$ increasingly diverge from $\mathrm{a}_{\mathrm{c}}$ and $C$ with range. The difference in the divergence between $M_{s}$ and $C$ for the two cases of $\beta=0.8$ and $\beta=1.2$ is associated with the different scattering characteristics in the Rayleigh and geometric regimes as discussed by Moate and Thorne (2009) and the increased attenuation feedback when $\beta=1.2$.

The results in Fig. 4 show the general instability of the iterative implicit inversion when the attenuation becomes an important component of the backscattered signal and illustrates the difficulty in obtaining accurate values for $a_{s}$ and $M_{s}$ under high attenuation conditions in marine studies, when the precise form of $f$ and $\chi$ are usually not known and have to be inferred using generic expressions based on formulations such a Eqs. (2) and (3). This is often the case for ABS studies conducted in energetic coastal waters where sediment variability leads to unavoidable uncertainties in the description of the intrinsic scattering properties of the suspended sediments and therefore inversions are necessarily based on generic expressions.

\section{B. Dual frequency inversion}

To try and overcome the feedback instabilities of the iterative implicit methodology, an approach proposed by Hurther et al. (2011) and known here as the dual frequency inversion is investigated. It has the major advantage that the calculated concentration at range $r$ from the transducer is independent of the concentration profile between the transducer and $r$, which removes the attenuation feedback instability inherent in the iterative implicit approach. However, the solution has the drawback that it requires the particle size distribution to be invariant with range and known. In the present work we first assess the impact of introducing variability in the sediment scattering characteristics into the basic dual frequency inversion, before extending the approach so that it does not require a priori knowledge of the particle size profile.

In the dual frequency approach a parameter $\Phi$ is defined using Eq. (1) as follows:

$$
\Phi=\frac{K \Re}{\Psi r} e^{-2 r \alpha_{w}}
$$

and hence we can write

$$
V^{2}=\Phi^{2} C e^{-4 \int_{0}^{r} \xi C d r} .
$$

Letting $J=V^{2} / \Phi^{2}$ and assuming an invariant particle size distribution with range we have

$$
J=C e^{-4 \xi \int_{0}^{r} C d r} .
$$

For a system with two frequencies Eq. (13) can be written for each frequency and expressed as

$$
\frac{J_{1}^{\xi_{2}}}{J_{2}^{\xi_{1}}}=C^{\xi_{2}-\xi_{1}} .
$$

The subscripts refer to the two frequencies. Rearranging to make $M_{s}$ the dependent variable and substituting for $\xi$ gives

$$
M_{s}=J_{1}^{\left(1-\chi_{1} / \chi_{2}\right)^{-1}} J_{2}^{\left(1-\chi_{2} / \chi_{1}\right)^{-1}} .
$$

The results for the dual frequency inversion are shown in Fig. 5(a) using the same representation for the lines, i.e., dashed for $\beta=0.8$ solid for $\beta=1$ and dotted for $\beta=1.2$. As in Fig. 4(a) using $\beta=1.0$ in the calculation of $V$ results in an inversion outcome identical with the input concentration profile used to calculate $V$. Using $\beta=0.8$ leads to an underestimate of the concentration and $\beta=1.2$ to an overestimate
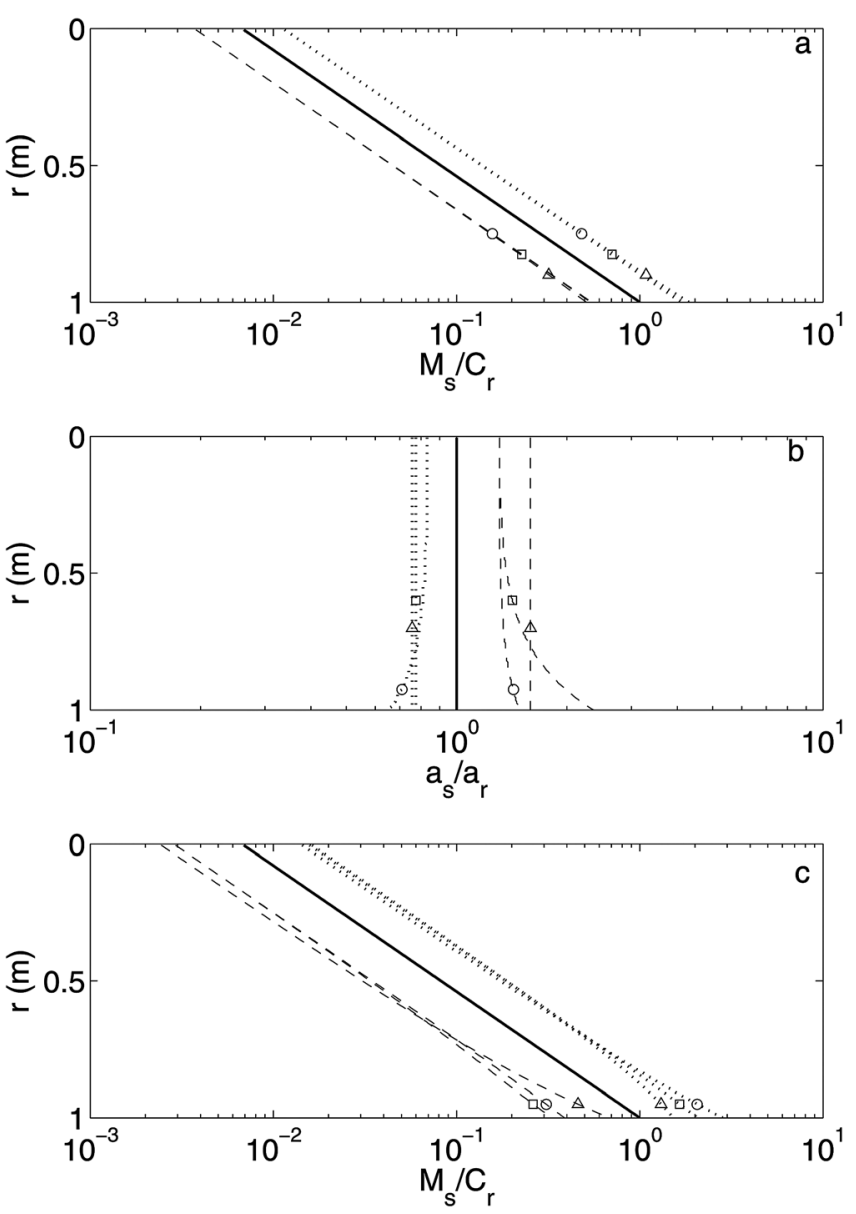

FIG. 5. (a) Profiles from the dual frequency inversion of the variation with range, $r$, of the normalized concentrations when the particle size was range independent and known. Profiles with range, $r$, from the hybrid constrained iterative implicit and dual frequency inversion of (b) the mean normalized particle size, $a_{s} / a_{r}$ and (c) the normalized concentration, $M_{s} / C_{r}$, when both mean particle size and concentration were unknown. The three lines are for $\beta=0.8(--), 1.0(-)$, and $1.2(\cdots)$, the symbols represent $C_{r}=1.0(\bigcirc), 5.0$ $(\square)$, and $20(\triangle) \mathrm{kgm}^{-3}$. 
of the concentration, as was the case in Fig. 4(a), however, what is clearly not present, is the increasing divergence between $M_{s}$ and $C$ with increasing range and reference concentration. Therefore the dual frequency inversion is much more stable than the iterative implicit inversion when $a_{s}$ is known and constant with range. Also because there is no need to iterate at each range bin to account for attenuation in the inversion, the dual frequency approach is computationally more efficient. The overhead to obtain this stability and speed is that a dual frequency system is required; however, this is not a serious limitation because many ABS operate with more than one frequency.

To extend the basic dual frequency approach to suspensions when the particle size is unknown a hybridization of the iterative implicit and dual frequency inversion was explored. In this approach the iterative implicit inversion was applied to obtain an estimate for $a_{s}$ using Eq. (10). However, to retain stability the iterative implicit inversion was restricted to the range $r_{s}$, where $\alpha_{s} r_{s}<0.2$ for the higher of the two frequencies, which in the present case was the $2.0 \mathrm{MHz}$ backscattered signal. The value of 0.2 was nominally optimum and is associated with a decrease in the backscattered signal intensity due to sediment attenuation of just over $50 \%$. The mean value of $a_{s}$ obtained from the iterative implicit inversion between $r=0-r_{s}$ was then used to evaluate the dual frequency inversion for $M_{s}$ over the whole profile. The results from this inversion are shown in Figs. 5(b) and 5(c). As previously the solid line represents $\beta=1$ in the calculation of $V$ and the inversion gives $a_{s}=a_{c}$ and $M_{s}=C$. Considering the particle size results shown in Fig. 5(b), the value for $a_{s}$ remain relatively consistent as the concentration increased for both $\beta=0.8$ and $\beta=1.2$. For the low concentration case, $C_{r}=1 \mathrm{kgm}^{-3}$, the size profiles shown in Fig. 4(b) and Fig. 5(b) are the same because the condition $\alpha_{s} r_{s}<0.2$ was met over the full range, $r=0-1.0 \mathrm{~m}$. However, as the concentration was increased the condition was exceeded and uniform particle size profiles result. These $a_{s}$ profiles are much more consistent with $a_{c}$ than those observed in Fig. 4(b). Using the $a_{s}$ profiles shown in Fig. 5(b) the dual frequency inversion was applied and the results for $M_{s}$ shown in Fig. 5(c) were obtained. The results for $\beta=0.8$ are comparable in Figs. 4(c) and 5(c) due to the condition $\alpha_{s} r_{s}<0.2$ not being exceeded for two of the concentrations and for the concentration where it is exceeded, $C_{r}=20 \mathrm{kgm}^{-3}$ the attenuation feedback was reduced because of the lower value of $\beta$. For the case where $\beta=1.2$ the feedback was enhanced, the condition $\alpha_{s} r_{s}<0.2$ was exceeded at 5 and $20 \mathrm{kgm}^{-3}$ leading to the constraint on $a_{s}$ being applied. This resulted in a significant improvement in the accuracy of the computed concentration profiles compared with those shown in Fig. 4(c).

It is readily acknowledged that having to use a hybrid approach when the particle size is unknown compromises to some degree the main advantage of the dual frequency approach, however, the combined constrained implicit dual frequency inversion is still a significant improvement over the more conventional unconstrained iterative implicit approach. Even if the constraint $\alpha_{s} r_{s}<0.2$ is applied to the calculation of $a_{s}$ in the iterative implicit solution, there is still the problem of positive feedback when concentrations are high, as shown

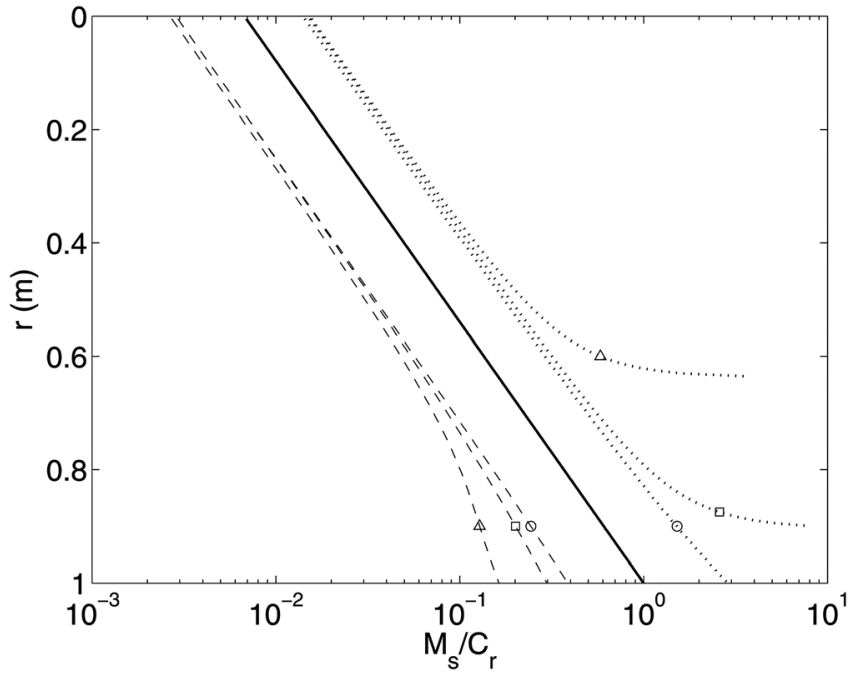

FIG. 6. Profiles of the variation with range, $r$, of the normalized concentration, $M_{s} / C_{r}$, from the constrained iterative implicit inversion when both the mean particle size and concentration were unknown. The three lines are for $\beta=0.8(--), 1.0(-)$, and $1.2(\cdots)$, the symbols represent $C_{r}=1.0(\bigcirc), 5.0$ $(\square)$, and $20(\triangle) \mathrm{kgm}^{-3}$.

by the resulting values for $M_{s}$ presented in Fig. 6 . This is particularly the case for $\beta=1.2$. As seen in Fig. 6 the output concentration profiles from a constrained iterative implicit solution, based on the size profiles shown in Fig. 5(b), underperforms relative to the hybrid solution shown in Fig. 5(c).

\section{Introduction of a size profile}

Invariably when using ABS to measure profiles of suspended sediment particle size and concentration in the bottom boundary layer, there will be sorting of sediment size with height above the bed. To represent this sorting, $a_{c}$ is given by the dashed line in Fig. 3(a) based on Eq. (5b) with $l=0.1 \mathrm{~m}$ and this profile was used to calculate $V$. The reduction of mean particle size by $40 \%$ from just above the bed to $1.0 \mathrm{~m}$ above the bed is consistent with observations (Thorne et al., 2002). Figure 7 shows the results for the dual frequency inversion using the same inputs as used for the inversions in Fig. 5, but with the particle size profile included in the calculation for the $V$ profiles. Only the results for $C_{r}=20$ $\mathrm{kgm}^{-3}$ are presented for brevity. Figure 7(a) shows the case when $a_{c}$ is known and only $M_{s}$ is being calculated. For the case when $\beta=1, M_{s}$ is no longer identical to $C$ represented by the crosses. There is a degree of divergence near the bed due to the introduction of a reduction in $a_{c}$ with height, which for the present case, gave a value for $M_{s}$ at $z_{r}$ of 28 $\mathrm{kgm}^{-3}$. Calculating the inversion with $\beta=0.8$ and 1.2 gives results comparable with those in Fig. 5(a) and substantially better than the results in Fig. 4(a). Therefore when there is size sorting with height above the bed, the dual frequency inversion overestimates the near bed concentration, however, this overestimate is small compared with the errors introduced by the iterative implicit inversion when $\beta \neq 1$. Similar results were obtained when a hybrid constrained iterative implicit and dual frequency inversion was carried out to obtain $a_{s}$ and $M_{s}$. The results are shown in Figs. 7(b) and 7(c). In this case $a_{s}=62 \mu \mathrm{m}$ and $M_{s}=33 \mathrm{kgm}^{-3}$ at $z_{r}$ when $\beta=1$; 

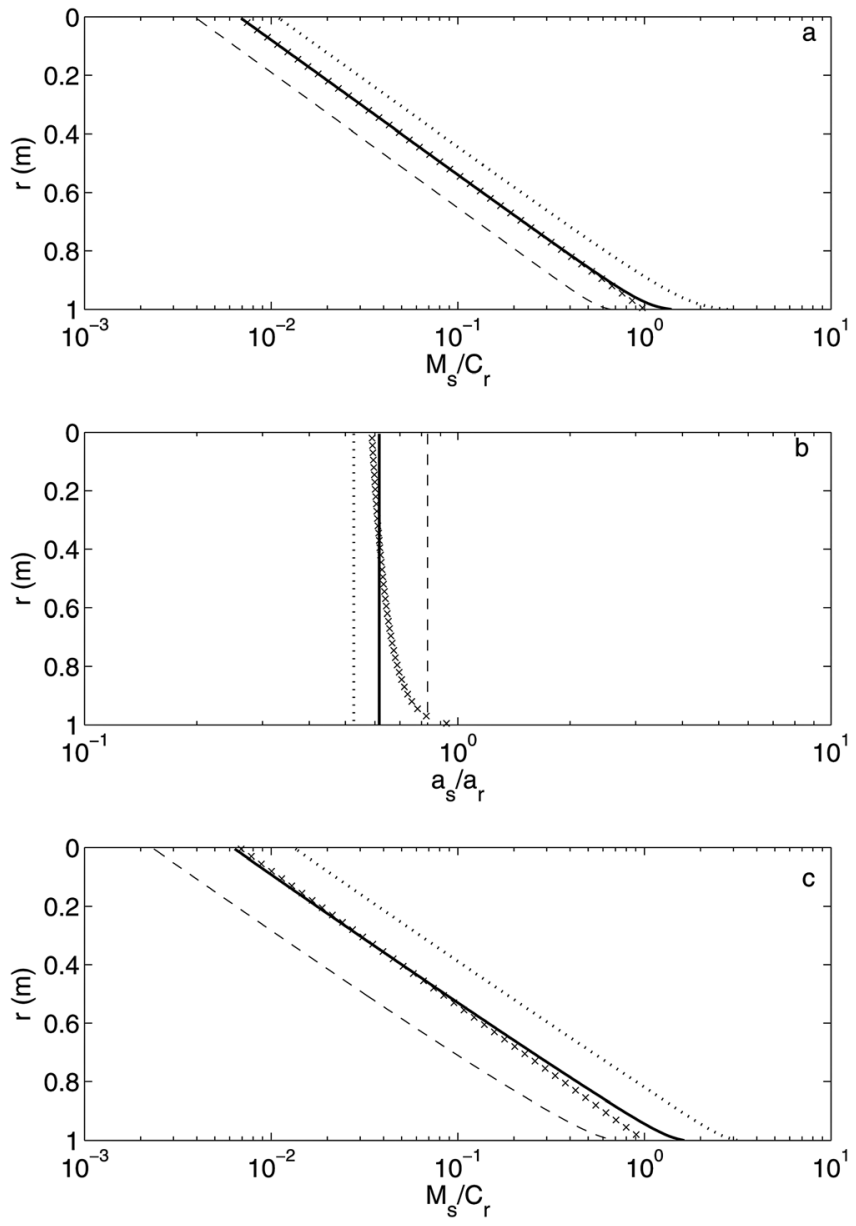

FIG. 7. (a) Profiles of the variation with range, $r$, of the normalized concentration, $M_{s} / C_{r}$, from the dual frequency inversion when the particle size was range dependent and known. Profiles of the variation with range, $r$, from the hybrid constrained iterative implicit and dual frequency inversion of; (b) the mean normalized particle size, $a_{s} / a_{r}$ and (c) the normalized concentration, $M_{s} / C_{r}$, when both the mean particle size and concentration were unknown. The three lines are for $\beta=0.8(--), 1.0(-), 1.2(\cdots)$ and $C_{r}=20 \mathrm{kgm}^{-3}$. The input profile for $a_{c}$ and $C$ are given by the symbol $(\times)$.

therefore near the bed the introduction of a particle size profile into the calculation of the backscattered signal resulted in an underestimate of the particle size and an overestimate of the concentration. Calculating the inversion with $\beta=0.8$ and $\beta=1.2$ leads to results which were significantly more consistent with $a_{c}$ and $C$ than using the unconstrained iterative implicit inversion results shown in Figs. 4(b) and 4(c). There is therefore a trade off when the particle size profile is a function of height above the bed. When $\beta=1$ the use of the dual frequency inversion will not precisely recover the input $a_{c}$ and $C$ profiles, however, when $\beta \neq 1$ and sediment attenuation is significant, the stability of the dual frequency inversion results in more accurate values of $a_{s}$ and $M_{s}$ than the iterative implicit inversion.

As discussed previously, in general the value of $\beta$ for a particular sediment will not be accurately known in marine studies. Therefore by varying $\beta$ an estimate for the error bars on the calculated $a_{s}$ and $M_{s}$ can be obtained with the constrained iterative implicit dual frequency inversion. This is a further advantage because such error bars would be difficult to calculate with an iterative implicit inversion because of the positive feedback of the errors which propagate and increase with range as shown in Figs. 4 and 6.

\section{Comparison on the inversions applied to laboratory data}

To assess the iterative implicit and dual frequency inversions experimentally, both methods were applied to 2.0 and 4.0 $\mathrm{MHz}$ backscatter measurements collected in a vertical sediment tower capable of generating a homogeneous suspension of quartz sand over a distance of $0.8 \mathrm{~m}$ (Moate and Thorne, 2009). The suspension had a particle size distribution, $n(a)$, which was lognormal, with $\sigma_{c} / a_{c}=0.4$. The concentration and particle size were uniform with range from the transducer, with $C=0.93 \mathrm{kgm}^{-3}$ and $a_{c}=50 \mu \mathrm{m}$ and the system constant $\mathfrak{R}$ had been measured (Betteridge et al., 2008). In this experimental case the intrinsic scattering properties of the sediments, $f_{i}$ and $\chi_{i}$, had been measured and were therefore accurately known (Moate and Thorne, 2009). This being the case it was therefore more logical to introduce the uncertainty in the scattering characteristics into the inversions calculations. Therefore the values for $f_{i}$ and $\chi_{i}$, associated with the different values of $\beta$ were applied to the inversions used to calculate $M_{s}$ and $a_{s}$ from $V$. To facilitate comparisons with the previous simulations, two inversions were carried out; (i) using simulated values of $V$ generated from the known $C$ and $a_{c}$ and (ii) using the measured values of $V$ backscattered from the laboratory suspension. The results from the simulation inversion are compared with the results from the inversion using the measured backscattered data.

First considered is the case when the known value for $a_{c}$ was used in the inversion and only $M_{s}$ was calculated. The iterative implicit inversion results for $M_{s}$ are shown in Fig. 8(a). The $4 \mathrm{MHz}$ data is shown to highlight the difficulties of the iterative implicit approach. Consistent with the simulations the dashed, solid, and dotted line were calculated using $\beta<1, \beta=1, \beta>1$. For the case of $\beta=1$ the original concentration profile is obtained in the simulation with $M_{s}=C$. For the cases of $\beta<1$ and $\beta>1$, four values for $\beta$ were used; respectively $\beta=0.90$ and $\beta=0.95$ and $\beta=1.05$ and $\beta=1.1$. The simulated results show values for $M_{s}$ that increasingly diverges from $C$ with range and changes in $\beta$. In Fig. 8(a) the simulations show $M_{s} / C_{r}>1$ for $\beta<1$ and $M_{s} / C_{r}<1$ for $\beta>1$. This trend in $M_{s} / C_{r}$ with $\beta$ is opposite to that shown in Fig. 4 , and due to the changes in $\beta$ being introduced into the inversion rather than into the calculation of $V$. The experimental results for the iterative implicit inversion obtained from the measured values of $V$ are shown by the open circles and the results are seen to be comparable with those of the simulations. The experimental inversions clearly illustrate the erroneous $M_{s}$ profiles obtained from the measured backscattered data, due to relatively small uncertainties in the values for $f_{i}$ and $\chi_{i}$, in the iterative implicit inversion. In Fig. 8(b) the results are shown for the dual frequency inversion. As seen in Fig. 8(b), both the simulations of $V$ and the measured values for $V$ give very comparable concentration profiles and in contrast to Fig. 8(a), neither show increasing divergence between $M_{s}$ and $C$ with range when $\beta \neq 1$. These results experimentally validate the stability of the basic dual frequency inversion when subject to uncertainties in $f_{i}$ and $\chi_{i}$. 

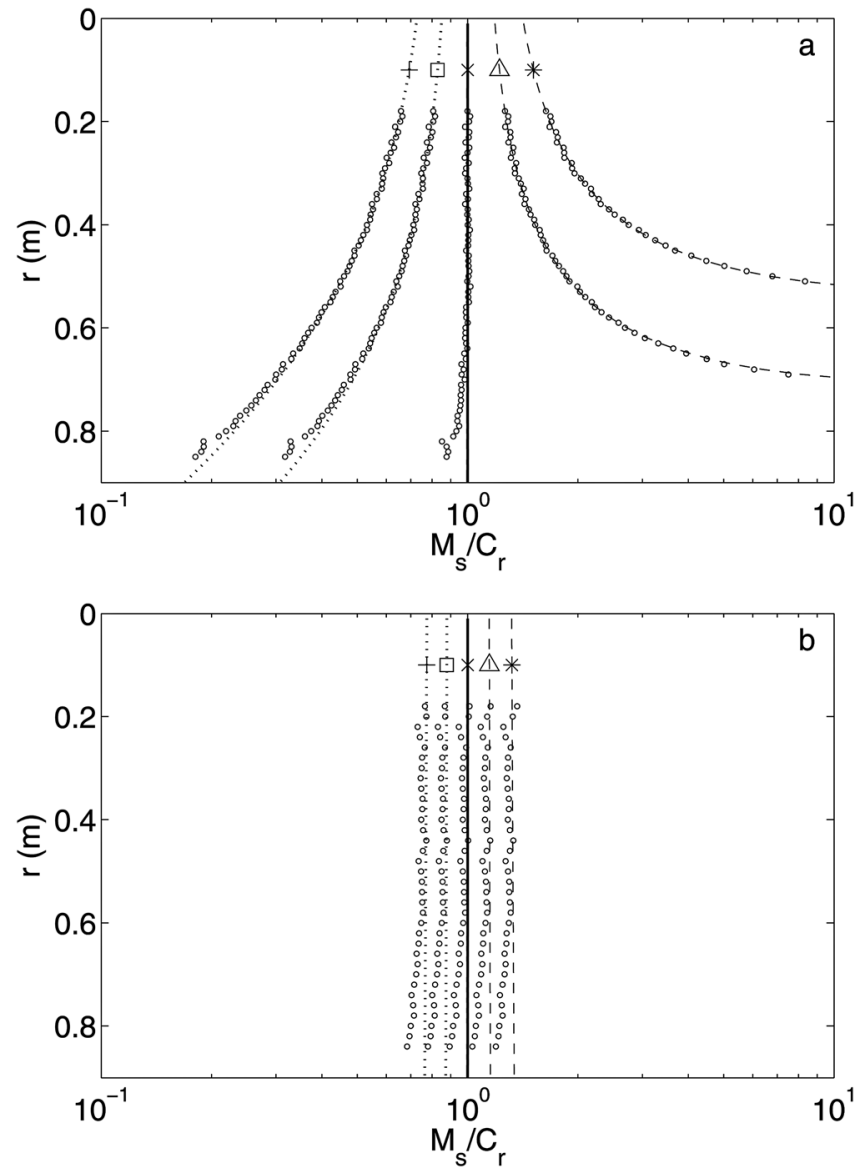

FIG. 8. Profiles of the variation with range, $r$, of the normalized concentration profiles, $M_{s} / C_{r}$, when the particle size was range independent and known. (a) Iterative implicit inversion and (b) dual frequency inversion. The lines are from simulated inversions and the open circles are from inversions using the measured backscattered signal. The symbol represent the different values for $\beta ; 0.9(*), 0.95(\triangle), 1.0(\times), 1.05(\square), 1.1(+)$.
Using the measured and simulated 2.0 and $4.0 \mathrm{MHz}$ backscattered $V$ profiles unconstrained iterative implicit and the hybrid constrained iterative implicit dual frequency inversions were carried out to obtain profiles of both $a_{s}$ and $M_{s}$. The results from the two inversions methodologies are shown in Fig. 9. The values for $\beta$ were $\beta=0.95,1.0$, and 1.05. Figures 9(a) and 9(b) show the results for the unconstrained $4.0 \mathrm{MHz}$ iterative implicit inversion using the measured and simulated values for $V$. The results show increasing divergence of both $a_{s}$ and $M_{s}$ from $a_{c}$ and $C$, respectively, with range when $\beta \neq 1$. The outputs again illustrate that uncertainties in the scattering parameters of the suspension can generate very erroneous particle size and concentration profiles using the iterative implicit approach. Conversely, the results in Figs. 9(c) and 9(d), calculated using the hybrid inversion, with the $\alpha_{s} r_{s}<0.2$ criterion applied at 4.0 MHz for the particle size calculation, clearly show much more stable results for both the measured and simulated backscattered signals, with no increasing divergence of $a_{s}$ and $M_{s}$ from $a_{c}$ and $C$, respectively, with range.

\section{E. Frequency selection and the impact of noise}

In the analysis presented above a ratio of two was used for the frequencies in the dual frequency inversions. This ratio was not specifically selected, the frequencies chosen were intended to reflect those used in ABS. However, it was considered of interest to examine how the choice of frequencies affects the dual frequency inversion. For this assessment some degree of noise has to be present, therefore the backscattered signals were modified as follows, $V_{i}^{2}=V_{i o}^{2}\left(1+\varepsilon \varphi_{i}\right)$, where $V_{i o}$ was the noise free value of $V_{i}, \varphi_{i}$ was a range profile of randomly generated numbers between -1.0 and +1.0 , $\varepsilon$ scaled the noise and had values between 0.01 and 0.2 and subscript $i=1,2$ represents the two frequencies with $i=1$
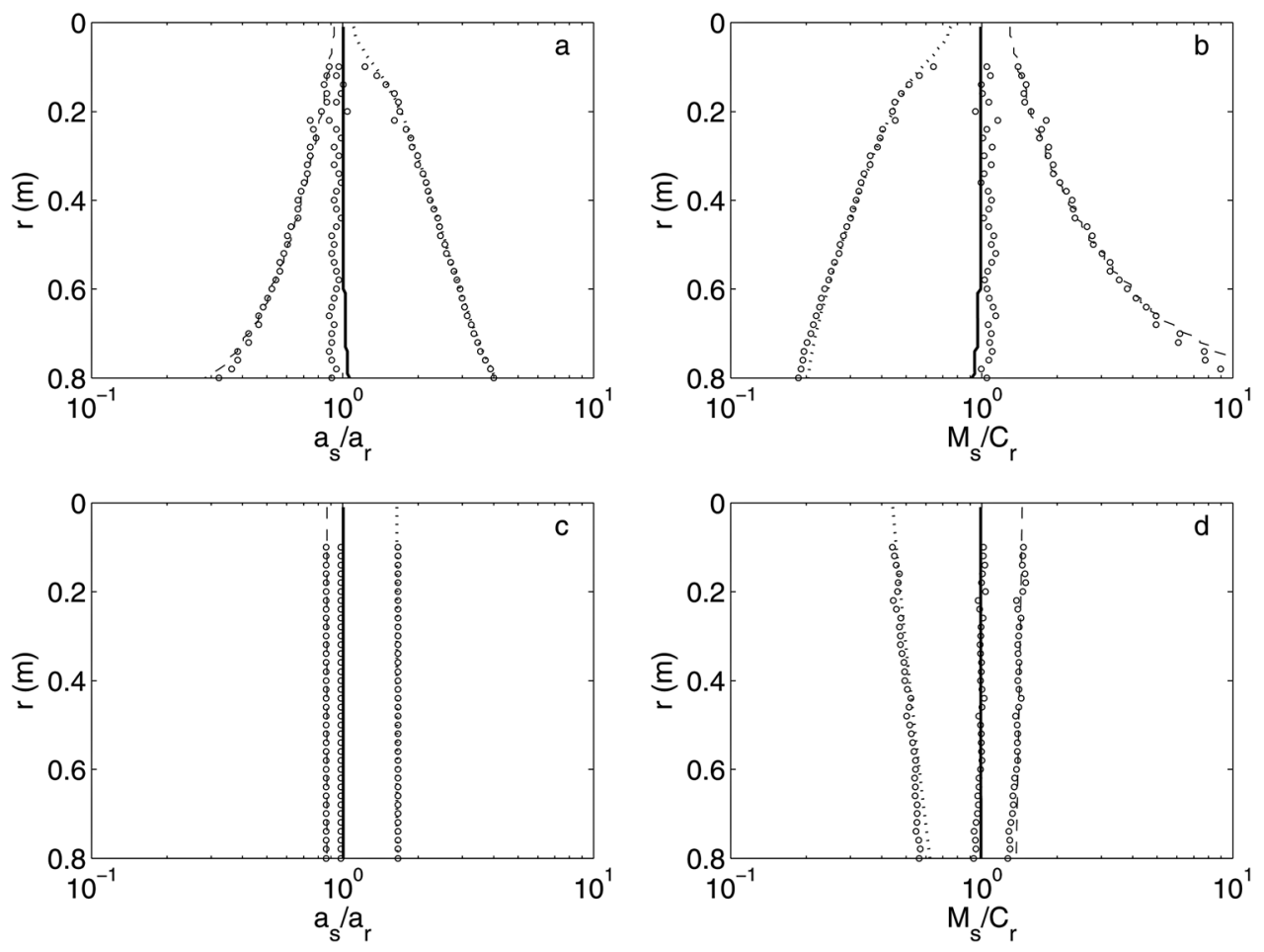

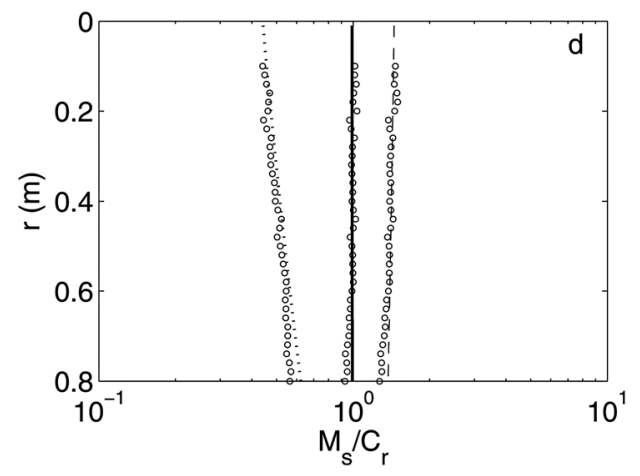

FIG. 9. Profiles of the variation with range, $r$, of the normalized mean particle size, $a_{s} / a_{r}$ and the normalized concentration, $M_{s} / C_{r}$. (a) and (b) were obtained using the iterative implicit inversion and (c) and (d) were obtained using the hybrid constrained iterative implicit dual frequency inversion. The lines are from simulated inversions and the open circles from inversions using the measured backscattered signal. The lines are for $\beta=0.95$ (--), $1.0(-), 1.05(\cdots)$. 


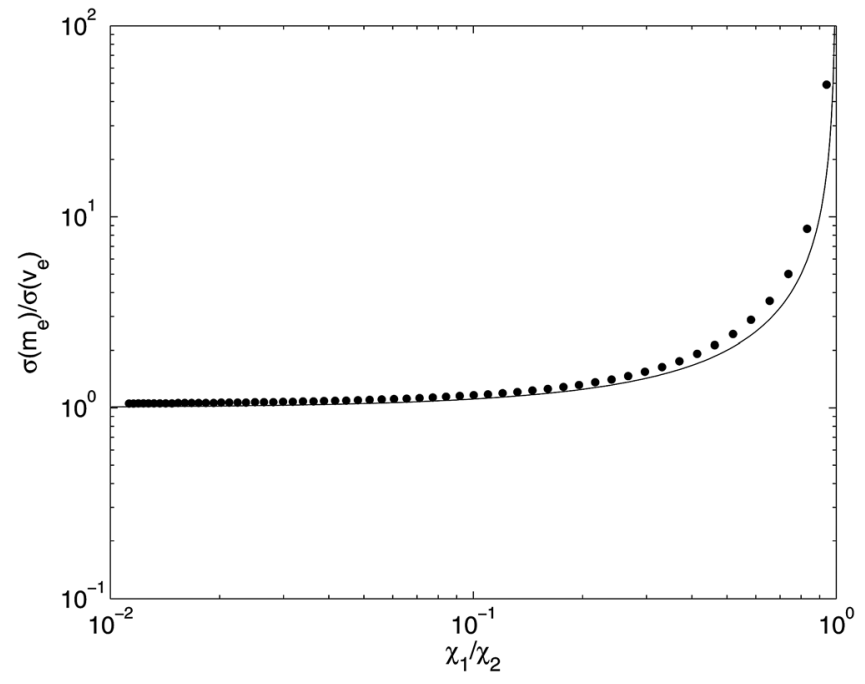

FIG. 10. Plot of the ratio of the concentration noise relative to the backscatter signal noise, $\sigma\left(m_{e}\right) / \sigma\left(v_{e}\right)$, with the ratio of the normalized total scattering cross sections, $\chi_{1} / \chi_{2}$. The solid circles were obtained using Eq. (15) and the solid line is given by $\left(1-\chi_{1} / \chi_{2}\right)^{-1}$.

being the lower frequency. Equation (15) was evaluated with the added noise and a non-dimensional generic curve was obtained which is shown by the solid circles in Fig. 10. The abscissa is given by $\chi_{1} / \chi_{2}$ and the ordinate is given by $\sigma\left(m_{e}\right) / \sigma\left(v_{e}\right)$, where $\sigma$ represents standard deviation and $m_{e}=\left(M_{s}-C\right) / C$ and $v_{e}=\left(V^{2}-V_{o}^{2}\right) / V_{o}^{2}=\varepsilon \varphi_{i}$. The result approximately followed the form $\left(1-\chi_{1} / \chi_{2}\right)^{-1}$ given by the solid line in the figure. The curve shows that as long as $\chi_{1} /$ $\chi_{2}<0.5, \sigma\left(m_{e}\right) / \sigma\left(v_{e}\right)$ is nominally uniform and comparable with unity, however, for $\chi_{1} / \chi_{2}>0.5 \sigma\left(m_{e}\right) / \sigma\left(v_{e}\right)$ rapidly increases with $\chi_{1} / \chi_{2}$. Therefore as long as the two frequencies used in the dual frequency inversion are sufficiently far apart that $\chi_{1} / \chi_{2}<0.5$, noise is not significantly amplified by the inversion. However, if the frequencies are so close together that $\chi_{1} / \chi_{2}>0.5$, or if both frequencies are approaching the geometric regime where $\chi_{1} / \chi_{2} \approx 1$, the variance in the concentration profile will be substantially greater than that of the backscattered signal. The results presented in Figs. 4-9 had a value of $\chi_{1} / \chi_{2} \approx 0.2$, therefore the introduction of noise into the dual frequency inversions gives nominally the same results as shown in the figures and the overall message about the advantages of using the dual frequency system is therefore the same. However, if the ratio of $\chi_{1} / \chi_{2}$ had been closer to unity, and some level of noise was present, the advantages of the dual frequency approach would not have appeared so clear and therefore consideration has to be given to the frequencies selected for a dual frequency system.

\section{DISCUSSION AND CONCLUSION}

The use of sound to measure boundary layer sediment processes is an ongoing developmental area in underwater acoustics, with its quantitative application having undergone significant advances over the past two decades. In the present study the use of sound to quantitatively measure profiles of suspended sediment particle size and concentration has been revisited. The use of multifrequency acoustic systems to measure these profiles has been reported in the literature for the past twenty years; however, there has been little analysis of the effectiveness of the commonly used iterative implicit inversions to accurately measure particle size and concentration.

Normally in marine applications of ABS, there will be uncertainties associated with the variable scattering characteristics of the sediments in suspension. These lead to errors in the profiles of the suspended sediment particle size and concentration derived from the acoustic data. This will be the case for any inversion methodology which employs generic scattering expressions, since the generic descriptions only approximate to the actual scattering characteristics of a particular suspension at a specific measurement site. The focus of the present paper has therefore been on how the particle size and concentration profiles are impacted by these scattering uncertainties and inversions employed.

To assess the impact the commonly used iterative implicit inversion was examined. This was carried out by introducing variability into the backscattered signal, $V$, through $\beta$ in the simulated study, and for the experiment case where $V$ was measured and the scattering characteristics known, the uncertainty was introduced through $\beta$ into the inversion. The iterative implicit solution was seen to work well for the case when sediment attenuation was weak, $C_{r}=1.0 \mathrm{~kg} \mathrm{~m}^{-3}$. However, as $C_{r}$ increased to $5.0 \mathrm{kgm}^{-3}$ and then $20.0 \mathrm{kgm}^{-3}$, attenuation became significant and errors arising from uncertainties in the scattering properties of the suspension accumulated and increased with range from the transducer. This led to profiles of particle size and concentration, which were substantially different from what was actually in suspension. The simulated and measured results presented here highlight the difficulties which can arise when using an iterative implicit inversion when sediment attenuation has a significant impact on the backscattered signal.

In an attempt to overcome the accumulative errors inherent in the iterative implicit inversion at high attenuation, a development using a dual frequency approach proposed by Hurther et al. (2011) has been examined. This is an explicit formulation with no requirement for iteration and has the significant advantage that the concentration calculated at one particular range is not dependent on calculations made between the transducer and the range of interest. When the precise conditions for the formulation are met, that is the particle size distribution is constant with range and known, the dual frequency approach is robust with regard to uncertainties in the scattering characteristics of the suspended sediments and substantially outperforms the iterative implicit inversion, both in terms of computation speed and the accuracy of the output concentration profiles. For calculations of particle size and concentration a hybrid constrained iterative implicit and dual frequency inversion was employed. This combined approach outperformed the iterative implicit inversion, even when it was also constrained using the $\alpha_{s} r<2$ criterion. However, it is acknowledged that having a hybrid approach when the particle size is unknown does add to the complication of the inversion. To relax the dual frequency restriction of having an invariant particle size with range, a particle size profile with height above the bed was introduced. The results showed that this did introduce errors into the calculated concentration profile if the particle 
size profile was known and into the size and concentration profile if both were unknown. However, as shown in Fig. 7, when a size profile is present and attenuation is significant, the errors in $M_{s}$ and $a_{s}$, due to the biases in the sediment scattering properties, were still substantially less using the dual frequency approach, than those generated by the iterative implicit inversions shown in Fig. 4. These results remained valid as noise was introduced into the backscattered signal, although it was necessary to keep $\chi_{1} / \chi_{2}<0.5$ to prevent the output noise of the inversion becoming excessive.

Although the work presented here was focused on the utilization of acoustics for boundary layer sediment process studies, the use of the dual frequency approach, subject to the incoherent scattering formulation which underpins the derivation of Eq. (15), should have application in other fields of acoustics where propagation attenuation is a problem. The technique may also have application in the field of electromagnetics when dual frequencies are available and the variation in the normalized total scattering cross section with $\mathrm{ka}$ can be explicitly expressed.

\section{ACKNOWLEDGMENTS}

This work was supported by NERC UK contracts FLOCSAM and FORMOST, LEGI-OSUG France, the SANDS component of the Integrating Activity HYDRALAB III, European Commission Contract No. 022441 (RII3) and the WISE component of the Integrating Activity HYDRALAB IV, European Commission Contract No. 261520.

Aydin, K., Zhao, Y., and Seliga, T. A. (1989). "Rain-induced attenuation effects on C-band dual-polarization meteorological radars," IEEE Trans. Geosci. Remote Sens. 27(1), 57-66.

Betteridge, K. F. E., Bell, P. S., Thorne, P. D., and Williams, J. J. (2006). "Evaluation of a triple axis Coherent Doppler Velocity Profiler for measuring near-bed flow: A field study," J. Atmos. Ocean. Technol. 23, 90-106.

Betteridge, K. F. E., Thorne, P. D., and Cooke, R. D. (2008). "Calibrating multi-frequency acoustic backscatter systems for studying near-bed suspended sediment transport processes," Cont. Shelf Res. 28, 227-235.

Bringi, V. N., Keenan, T. D., and Chandrasekar, V. (2001). "Correcting Cband radar reflectivity and differential reflectivity data for rain attenuation: A self-consistent method with constraints," IEEE Trans. Geosci. Remote Sens. 39(9) 1096-1915.

Cheel, R. A., and Hay, A. E. (2008). "Cross-ripple patterns and wave directional spectra," J. Geophys. Res.[Oceans] 113, C10009.

Crawford, A. M., and Hay, A. E. (1993). "Determining suspended sand size and concentration from multifrequency acoustic backscatter," J. Acoust. Soc. Am. 94(6), 3312-3324.

Davies, A. G., and Thorne, P. D. (2005). "Modelling and measurement of sediment transport by waves in the vortex ripple regime," J. Geophys. Res. 110, C05017, doi:1029/2004JC002468, 2005.

Dohmen-Janssen, C. M. and Hanes, D. M. (2005). "Sheet flow and suspended sediment due to wave groups in a large wave flume," Cont. Shelf Res. 25(3), 333-347.

Foote, G. F. (1990). "Correcting acoustic measurements of scatter density for extinction" J. Acoust. Soc. Am. 88(3), 1543-1546.

Foote, K. G. (1992). "Determining the extinction cross section of aggregating fish," J. Acoust. Soc. Am. 91(4), 1983-1989.

Foote, K. G. (1999). "Extinction cross-section of Norwegian spring-spawning herring," ICES J. Mar. Sci. 56, 606-612.

Hanes, D. M., Vincent, C. E., Huntley, D. A., and Clarke, T. L. (1988). "Acoustic measurements of suspended sand concentration in the $C^{2} S^{2}$ experiment at Stanhope Land, Prince Edwards Island," Mar. Geol. 81, $185-196$.
Hatcher, A., Hill, P., Grant, J., and Macpherson, P. (2000). "Spectral optical backscatter of sand in suspension: effects of particle size, composition and colour," Mar. Geol. 168, 115-128.

Hay, A. E., and Sheng, J. (1992). "Vertical profiles of suspended sand concentration and size from multifrequency acoustic backscatter," J. Geophys. Res. 97(C10), 15661-15677.

Holdaway, G. P., and Thorne, P. D. (1997). "Determination of a fast and stable algorithm to evaluate suspended sediment parameters from high resolution acoustic backscatter systems," Seventh International Conference on Electronic Engineering in Oceanography, Southampton Oceanography Centre, UK, 23-25 June, pp. 86-92.

Hurther, D., and Lemmin, U. (2008). "Improved turbulence profiling with field adapted acoustic Doppler velocimeters using a bi-frequency Doppler noise suppression method," J. Atmos. Ocean. Technol. 25(2), 452-463.

Hurther, D., Thorne, P. D., Bricault, M., and Lemmin, U. (2011). "A multifrequency Acoustic Concentration and Velocity Profiler for boundary layer measurements of fine-scale flow and sediment transport processes," Coastal Eng. DOI: 10.1016/j.coastaleng. 2011.01.006.

Hurther, D., and Thorne, P. D. (2011). "Acoustic measurements of velocity and sediment concentration within the suspension and bedload layers above a migrating sand ripple under regular asymmetric waves," Proceeding of Coastal Sediments 2011, Miami, Florida, 2-6 May (World Scientific, Singapore), pp. 1641-1650.

Lee, G. H., Dade, W. B., Friedrichs, C. T, and Vinceny, C. E. (2004). "Examination of reference concentration under waves and currents on the inner shelf," J. Geophys. Res. [Oceans] 109, C02021.

Lee, T. H., and Hanes, D. M. (1995). "Direct inversion method to measure the concentration profile of suspended particles using backscattered sound," J. Geophys. Res. 100(C2) 2649-2657.

Marzoug, M., and Amayenc, P. (1994). "A class of single and dual frequency algorithms for rain-rate profiling from spaceborne radar. Part I: Principles and tests from numerical simulations," J. Atmos. Ocean Technol. 11, 1480-1506.

Moate, B. D., and Thorne, P. D. (2009). "Measurements and inversion of acoustic scattering from suspensions having broad size distributions," J. Acoust. Soc. Am. 126(6), 2905-2917.

Nielsen, P. (1986). "Suspended sediment concentration under waves," Coastal Eng. 10, 23-31.

Schaafsma, A. S., and Hay, A. E. (1997). "Attenuation in suspensions of irregularly shaped sediment particles: A two-parameter equivalent spherical scatterer model," J. Acoust. Soc. Am. 102(3), 1485-1502.

Shen, C., and Lemmin, U. (1996). "Ultrasonic measurements of suspended sediments: A concentration profiling system with attenuation compensation,” Meas. Sci. Technol. 7(9), 1191-1194.

Shen, C., and Lemmin, U. (1998). "Improvements in acoustic sediment concentration profiling using an LMS compensation algorithm," IEEE J. Ocean. Eng. 23(2), 96-104.

Sheng, J., and Hay, A. E. (1988). "An examination of the spherical scatterer approximation in aqueous suspensions of sand," J. Acoust. Soc. Am. 83, 598-610.

Soulsby, R., (1997). Dynamics of Marine Sands (Thomas Telford, London), 249 pp.

Smyth, C., Hay, A. E., and Zedel, L. (2002). "Coherent Doppler Profiler measurements of near-bed suspended sediment fluxes and the influence of bed forms," J. Geophys. Res. 107, 3105.

Snyder, J. C., Bluestein, H. B., and Zhang, G. (2010). "Attenustion correction and Hydrometeor classification of high resolution X-band dual polarized mobile radar measurements in severe convective storms," J. Atmos. Ocean. Technol. 27, 1979-2001.

Thorne, P. D., Vincent, C. E., Hardcastle, P. J., Rehman, S., and Pearson, N. (1991). "Measuring suspended sediment concentrations using acoustic backscatter devices," Mar. Geol. 98, 7-16.

Thorne, P. D., Hardcastle, P. J., and Soulsby, R. L. (1993). "Analysis of acoustic measurements of suspended sediments," J. Geophys. Res. 98, 899-910.

Thorne, P. D., Holdaway, G. P., and Hardcastle, P. J. (1995). "Constraining acoustic backscatter estimates of suspended sediment concentration profiles using the bed echo," J. Acoust. Soc. Am. 98(4), 2280-2288.

Thorne, P. D., and Hardcastle, P. J. (1997). "Acoustic measurements of suspended sediments in turbulent currents and comparison with in-situ samples," J. Acoust. Soc. Am. 101(5), (Pt. 1), 2603-2614.

Thorne, P. D., and Hanes, D. M. (2002). "A review of acoustic measurement of small-scale sediment processes," Cont. Shelf Res. 22, 603-632.

Thorne, P. D., Williams, J. J., and Davies, A. G. (2002). "Suspended sediments under waves measured in a large scale flume facility," J. Geophys. Res. 107(C8), 4.1-4.16. 
Thorne, P. D., and Buckingham, M. J. (2004). "Measurements of scattering by suspensions of irregularly shaped sand particles and comparison with a single parameter modified sphere model," J. Acoust Soc Am. 116(5), 2876-2889.

Thorne, P. D., Agrawal, Y. C., Cacchione, D. A. (2007). "A comparison of near-bed acoustic backscatter and laser diffraction measurements of suspended sediments," IEEE J. Ocean. Eng. 32(1), 225-235.

Thorne, P. D., and Meral, R. (2008). "Formulations for the scattering properties of sandy sediments for use in the application of acoustics to sediment transport," Cont. Shelf Res. 28, 309-317.

Thorne, P. D., and Bell, P. S. (2009). "Acoustic measurement of near-bed sediment transport processes," in Encyclopedia of Ocean Sciences, 2nd ed., edited by J. H Steele, S. A Thorpe, and K. K. Turekian (Academic, New York), pp. 3416-3429.

Thosteson, E. D., and Hanes, D. M. (1998). "A simplified method for determining sediment size and concentration from multiple frequency acoustic backscatter measurements," J. Acoust. Soc. Am. 104(2), 820-830.

Toresen, R. (1991). "Absorption of acoustic energy in dense herring schools studied by the attenuation in the bottom echo signal," Fish. Res. 10, 317 327.

Traykovski, P. (2007). "Observations of wave orbital scale ripples and a nonequilibrium time-dependent model," J. Geophys. Res. [Oceans] 112, C06026

van der Werf, J. J. Ribberink, J. S., O’Donoghue T., and Doucette, J. S. (2006). "Modelling and measurement of sand transport processes over full-scale ripples in oscillatory flow," Coastal Eng. 53, 657-673.

van der Werf, J. J., Magar, V., Malarkey, J., Guizien, K., and O’Donoghue, T. (2008). "2DV modelling of sediment transport processes over full-scale ripples in regular asymmetric oscillatory flow," Cont. Shelf Res. 28(8), 1040-1056.

Van Rijn, L. C. (1993). Principles of Sediment Transport in Rivers, Estuaries and Coastal Seas (Aqua Publications, Amsterdam, The Netherlands), $633 \mathrm{pp}$.

Vincent, C. E., and Green, M. O. (1990). "Field-measurements of the suspended sand concentration profiles and fluxes and of the resuspension coefficient gamma over a rippled bed," J. Geophys. Res. 95(C7), 1159111601.

Vincent, C. E., Hanes, D. M., and Bowden, A. J. (1991). “Acoustic measurements of suspended sand on the shoreface and the control of concentration by bed roughness," Mar. Geol. 96, 1-18

Vincent, C. E., (2007). "Measuring suspended sand concentration using acoustic backscatter: a critical look at the errors and uncertainties," in, Coastal and Shelf Sediment Transport, Geological Society of London, Special Publications, edited by P.S. Balson and M.B. Collins (The Geological Society, London), Special Publication No. 274, pp. 7-15.

Williams, J. J., Bell, P. S., Thorne, P. D., Metje, N., and Coates, L. E. (2004). "Measurements and prediction of wave-generated suborbital ripples," J. Geophys. Res. 109, 1-18, CO2004.

Young, R. A., Merrill, J. T., Clarke, T. L., and Proni, J. R. (1982). "Acoustic profiling of suspended sediments in the marine bottom boundary layer," Geophys. Res. Lett. 9, 175-178.

Zedel, L., and Hay, A. E. (2002). "A three component bistatic coherent Doppler velocity profiler: Error sensitivity and system accuracy,” IEEE J. Ocean. Eng. 27, 717-725.

Zhao, X., and Ona, E. (2003). "Estimation and compensation models for the shadowing effect in dense fish aggregation,” ICES J. Mar. Sci. 60, 155-163. 